\title{
Tyrosine phosphatases in the HER2-directed motility of ovarian cancer cells: Involvement of PTPN12, ERK5 and FAK
}

\author{
Emma Villa-Moruzzi \\ Department of Experimental Pathology, via Roma 55, University of Pisa, 56126 Pisa, Italy \\ Tel.: +39050 2218 551; Fax: +390502218557 \\ E-mail:emma.villa@med.unipi.it
}

\begin{abstract}
Background: HER2 activation in tumours supports multiple signalling pathways, including those regulating invasion and metastasis. Among the involved genes, Tyrosine and Dual Specificity Phosphatases (PTPs and DSPs) may play a relevant, though not completely clear role.

Methods: HER2 was silenced in ovarian SKOV-3 cells, a genome-wide expression analysis of PTPs and DSPs was performed, the effects on cell motility were analysed and compared with those of PTPN12-silencing, focusing on FAK.

Results: HER2-silencing altered the expression of 4 PTPs and 6 DSPs; PTPN12 displayed also 3-4-fold protein increase. Conversely, PTPN12-silencing enhanced migration, suggesting that PTPN12 down-modulation by HER2 favours motility. HER2-silencing inactivated FAK, in quiescent and migrating cells, involving FAK dephosphorylation at Y397 and S910. Conversely, in PTPN12-silenced cells FAK activity was close to control, altogether suggesting that PTPN12 targets Y397. As regards to S910, cell-treatment with the MEK inhibitor UO126 and ERK5-silencing indicated its targeting by ERK5. Loss of pS910 and decreased ERK5 kinase activity in HER2-silenced cells confirmed their control by HER2.

Conclusions: The results indicate the contribution of PTPN12, targeting FAK Y397, and ERK5, targeting FAK S910, to the HER2-driven cell motility, thus depicting new aspects of the complex cross-talk between HER2 and the motility machinery.
\end{abstract}

Keywords: Tyrosine phosphatase, HER2, protein phosphorylation, cell migration

\section{Introduction}

The HER2 receptor controls cell proliferation and motility [1-3] and its mutations or amplification have been related to solid tumours, including breast, ovary, lung, prostate and colon cancers $[4,5]$. The role of HER2 in ovary cancer is less understood [6]. However, poor prognosis is linked to tumour spreading beyond the ovaries, an event requiring enhanced motility. Since HER2 controls motility, it is conceivable that its upregulation contributes to motility in ovary cancer cells. HER2 up-regulation is accompanied by alteration of a number of genes, including protein kinases [1]. Conversely, few data exist on Tyrosine phosphatases (PTPs) and Dual-Specificity phosphatases (DSPs) and the existing studies generally focused on posttranslational regulation [7], as we discussed elsewhere [8].
Thirty eight PTPs, 21 receptor and 17 soluble, and approximately 65 DSPs are found in the human genome. DSPs include MKPs, SSH, PTP4A, cdc14, PTEN, Myotubularins, CDC25 and EYA. PTPs and DSPs contribute to control cell proliferation, adhesion and migration, differentiation and survival [7, 9]. They may not only terminate the action of protein kinases, hence behaving like anti-oncogenes (e.g., the DUSPs/MKPs that dephosphorylate MAPKs [10]) but also transduce positive signals, likewise the case of PTPN11 [11], PTPN1 [12], PTPRE [13] and cdc25 [14]. In the HER2 signaling, negative regulation was reported for PTPN18 [15], whereas a favoring role was documented for PTPN11 [16] and PTPN1 [17]. Little is known of their role in ovary cancer, particularly in the HER2-overexpressing type, highly motile at some stages [6]. It is conceivable that the PTPs and DSPs 
present in motility pathways may have a role in the motility sustained by HER2.

PTPN12 (PTP-PEST) [18] is a critical regulator of cell adhesion, migration and cell-cell junction, interacting with cytoskeletal and signalling proteins, such as small GTPases, FAK, CAS, paxillin, src, abl, and others [19-22]. PTPN12 generally behaves like a negative regulator of motility, but in ras-activated cells it was also reported to promote migration through the inhibition of FAK [23]. Several aspects of the PTPN12 mechanism are still unclear, particularly concerning its substrates in different cell types. For instance, src, a PTPN12 substrate in migration and growth factor signalling, is activated by PTPN12 in osteoclasts [24] and inactivated in intestinal cells [25].

FAK is both an enzyme and a platform which recruits signalling molecules to the focal adhesions [26-28], and is often altered in cancer cells [29]. During migration FAK undergoes cycles of activation/inactivation and changes at its multiple phosphorylation sites [26, 28]. Several PTPs and some DSPs are regulated during migration [9]. In addition to PTPN12, studies indicated roles for PTPN11 [11, 25] and PTPD1 [30] in the regulation of FAK and src. In past investigations we found that FAK S910 was phosphorylated by ERK5 and involved in cell shaping and motility [31], whereas FAK S722 was phosphorylated by GSK3- $\beta$ during fibroblasts adhesion and late migration [32]. Both sites were dephosphorylated by the PP1 $\delta$ isoform $[31,32]$.

Several members of the MAP kinase family [33] regulate motility [28, 34]. ERK1/2 was reported to phosphorylate FAK S910 in fibroblasts, in response to growth factors and stress stimuli [35] or ras activation [23]. Conversely, we found that FAK S910 was phosphorylated by ERK5 in cells stimulated with phorbol ester [31]. More recently the involvement of ERK5 in motility was further supported by its activation in integrin-mediated epithelial cell attachment [36], keratinocyte migration [37] and focal contact turnover [38]. Generally ERK5 is activated downstream of receptor tyrosine kinases [39], including HER2 in breast cancer cells [40].

This study investigates the expression of PTPs and DSPs in SKOV-3 cells, a highly motile, HER2-overexpressing ovary carcinoma cell line. HER2 was silenced and the expression analysis adopted a DNA microarray, targeting all the PTPs and DSPs of the human genome [8]. PTPN12, displaying the most significant alteration, was further analysed. The results identified: a) PTPN12 as a negative regulator of
SKOV-3 cell motility, through the control of FAK phosphorylation at Y397 and its down-modulation by HER2, and b) the HER2-dependent regulation of FAK phosphorylation at S910 through the activation of ERK5, altogether unravelling new aspects of the complex cross-talk between HER2 and the motility machinery.

\section{Materials and methods}

\subsection{Antibody sources}

Antibodies were purchased as follows: FAK, pY576/577 of FAK, src, PTPN6 (SHP1) and PTEN (Santa Cruz Biotech); pS722, pS910 and pY397 of FAK (BioSource); HER2, ERK5, PTPN12 (PTPPEST), AKT, pS473 of AKT and pY418 of src (Cell Signaling Biotech); ERK1/2, pERK1/2 (pT202/pY204 of ERK1 and pT185/pY187 of ERK2) and $\alpha$ tubulin (Sigma Aldrich); PTPRK (Abcam); PTPN11 (SHP2) (Upstate). The anti-CDC25C antibodies were produced as described [41].

\subsection{Cell culture, treatment and extract}

SKOV-3 cells (originally from ATCC and re-characterized at the Istituto Nazionale Tumori, Milan, Italy) were grown in RPMI added with $10 \%$ fetal calf serum (Sigma Aldrich) [32]. In one experiment subconfluent cells were exposed to the MEK inhibitor UO126 (Calbiochem), diluted in DMSO, or to DMSO alone (control). For extraction, cells were washed twice in cold PBS and lysed in $50 \mathrm{mM}$ TRIS-HCl, $\mathrm{pH} 7.5,150 \mathrm{mM} \mathrm{NaCl}, 5 \mathrm{mM}$ EDTA, $1 \%$ Triton X100, 7.5 mM 2-mercaptoethanol (lysis buffer), added with $1 \mathrm{mM}$ orthovanadate, $50 \mathrm{mM} \mathrm{NaF}$ and protease inhibitors $(0.02 \%$ benzamidine, $0.02 \%$ PMSF, $0.02 \%$, TPCK, $10 \mu \mathrm{g} / \mathrm{ml}$ soybean trypsin inhibitor and $4 \mu \mathrm{g} / \mathrm{ml}$ leupeptine), followed by centrifugation at $4{ }^{\circ} \mathrm{C}$ and immediate use or storage at $-80^{\circ} \mathrm{C}$.

\subsection{RNAi silencing}

Cells were transfected with the shRNAmir vectors targeting human HER2 or PTPN12, or with the empty vector, as control (Open Biosystems), using Lypophectamine 2000 as transfection reagent (Invitrogen). Cells were grown and selected in the presence of $5 \mu \mathrm{g} / \mathrm{ml}$ 
puromycin starting from $24 \mathrm{~h}$ after transfection. Cells were further grown and subcloned by dilution in the presence of puromycin and analyzed for the presence of the targeted protein by immunoblotting; cells displaying sub-total gene silencing were further grown and used in the experiments.

\subsection{DNA array}

Custom oligo GEArray, displaying 60-mer oligonucleotide probes, targeting 103 PTPs and DSPs and 5 housekeeping genes (RPS27A, GAPDH, B2 M, HSP90AB1 and ACTB) were produced by SABiosciences. cRNA $(2 \mu \mathrm{g})$ was used for overnight hybridization, followed by peroxidase-based chemiluminescence detection on X-ray films, according to the manufacturer 's instructions. Data extraction, normalization with the indicated housekeeping genes and analysis used the GEArray Expression Analysis Suite (SABiosciences). Expression levels are given as the ratios of treated to control cells. To boost the results reliability, only changes greater than $30 \%$ were reported [8].

\subsection{Cell proliferation assay}

Cells were seeded as specifically indicated, grown for $48 \mathrm{~h}$, washed in PBS, fixed in 3\% paraformaldehyde and $2 \%$ sucrose in PBS, pH 7.5, for $10 \mathrm{~min}$ at room temperature, stained with Crystal Violet and lysed in $10 \%(\mathrm{v} / \mathrm{v})$ acetic acid. Optical density of the lysate was then measured at $595 \mathrm{~nm}$.

\subsection{In vitro cell migration into a wounded area and transwell assay}

The scratch-wound and transwell assays were performed as described previously [32]. Migration was assessed on stained cells by calculating the percentage of wound closure (i.e., the distance moved by the cells) with respect to the $100 \%$ open wound (time 0 ).

\subsection{Immunoprecipitation, electrophoresis and immunoblotting}

Immunoprecipitation with Protein A- or Protein G-Sepharose (Sigma Aldrich), electrophoresis and immunoblotting were performed as described previously [32]. For immunoblot quantification, the PTP or
DSP protein bands from treated and control cells were analyzed by densitometric scanning and normalized to the $\alpha$-tubulin band analyzed in the same experiment (i.e., the PTP or DSP/ $\alpha$-tubulin value ratios were calculated). Normalized data from different experiments were averaged, as specifically indicated. Results are given as percent of averages from control cells.

\subsection{In-gel tyrosine phosphatase assay}

The phosphatase activity of cell lysates or associated with immunoprecipitated FAK was assayed using the substrate ${ }^{32} \mathrm{P}$-labeled poly-Glu-Tyr, introduced into a $10 \%$ polyacrylamide-SDS gel [42]. The gel was immediately used to run the samples (mixed with Laemmli buffer). This was followed by fixing, phosphatase renaturation and substrate dephosphorylation during overnight incubation, as indicated by the original method [43]. Subsequently, the gel was stained with Coomassie brilliant blue, dried and subjected to autoradiography to detect active phosphatases, indicated by dephosphorylated areas of discrete Mr. PTPN12 identification was based on: a) the $\mathrm{Mr}$ of the active phosphatase (around $120 \mathrm{k}$ ), a broad band, due to degradation of its PEST-sequence [21]; b) immunodetection of PTPN12 in samples run in parallel with the gel for the activity assay (see Fig. 4A); c) band enrichment in anti-PTPN12 immunocomplexes (not shown).

\subsection{ERK5 and FAK kinase assays}

ERK5 was assayed using either the ERK5-specific substrate Mef2, as described previously [31, 44] or myelin basic protein (MBP). For MBP kinase, ERK5 was first immunoprecipitated from $0.5 \mathrm{mg}$ of cell extract, additionally washed with $25 \mathrm{mM}$ imidazole, $\mathrm{pH} 7.5$, and incubated with $20 \mu \mathrm{g}$ MBP at $30^{\circ} \mathrm{C}$ for $15 \mathrm{~min}$. All the rest was as indicated for Mef2 kinase, apart from control assays, that were either prepared with unrelated IgGs or without MBP. FAK was assayed following immunoprecipitation, as described previously [32]. The reaction, containing $2.5 \mathrm{mg} / \mathrm{ml}$ poly-Glu-Tyr (Sigma Aldrich), $0.6 \mu \mathrm{M}$ PP2 (src inhibitor, Tocris) and $0.5 \mathrm{mM}$ orthovanadate (all the rest was as indicated for the MBP kinase) was run for $20 \mathrm{~min}$ at $30^{\circ} \mathrm{C}$ and terminated by spotting $40 \%$ of the mix on Whatman P81 chromatography paper $(2 \times 1 \mathrm{~cm})$, followed by extensive washing in $10 \mathrm{mM}$ 
$\mathrm{H}_{3} \mathrm{PO}_{4}$ and counting in a $\beta$-scintillation counter. The kinase activity was calculated after subtracting the cpm of control immune complexes, prepared with unrelated IgGs.

\section{Results}

\subsection{HER2 sustains cell motility}

To find if HER2 sustains motility, we prepared SKOV-3 cells carrying stable HER2-silencing. Such cells displayed about $70 \%$ decrease in HER2 protein with respect to control cells and decrease in the activity of two members of HER2 signalling, ERK1/2 and AKT, based on the marked decrease in their phosphorylated active forms (pERK1/2 and pAKT, Fig. 1A). Concomitantly, also cell proliferation decreased (measured at $48 \mathrm{~h}$ from cell plating, Fig. 1B). HER2 silencing impaired migration, whether assessed through wound-healing assay (Fig. 1C) or trans-well assay (Fig. 1D).

Although both migration results are bound to be influenced by the decreased proliferation, the effect of HER2 silencing on migration was so marked (see e.g., $12 \mathrm{~h}$, Fig. 1C) that cannot be explained by the mere decrease in cell number. Altogether, the results indicated that HER2 contributed to sustain SKOV-3 cell motility. The subsequent results, indicating the

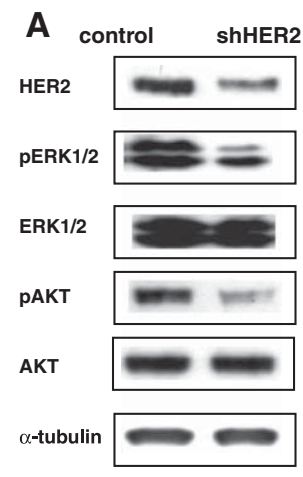

C

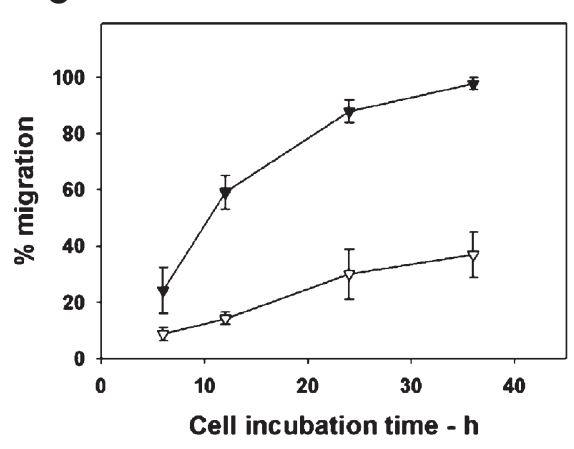

B

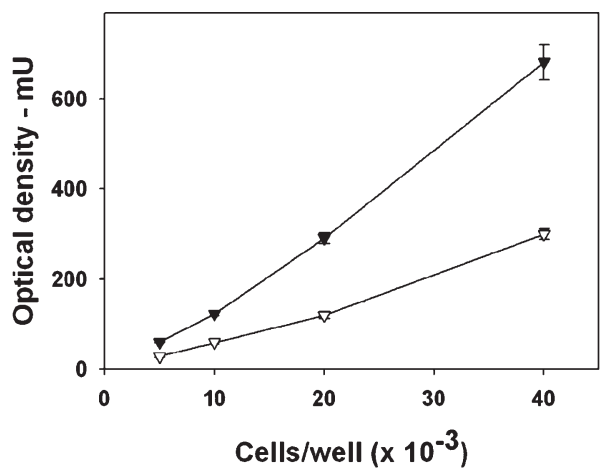

D

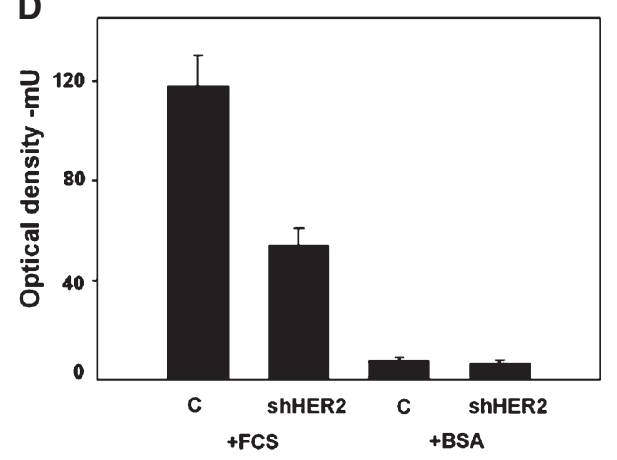

Fig. 1. HER2-silencing and effects on the migration of SKOV-3 cells. (A) HER2 and its signaling. Extracts were prepared from cells carrying stable HER2 silencing and control cells (carrying the empty vector) and used to immunodetect HER2, pERK1/2 (pT202/pY204 of ERK1 and pT185/pY187 of ERK2), ERK1/2, pAKT (pS473), AKT and $\alpha$-tubulin. pERK1/2 and pAKT detect the active form of the enzymes. The data are representative of several independent experiments. (B) Cell proliferation assay. The indicated amounts of cells were seeded in 24-well Costar plates, grown for $48 \mathrm{~h}$, fixed, stained with Crystal Violet, lysed and analyzed spectrophotometrically. Average of 4 experiments, using 2 different clones of HER2-silenced (open symbols) and control (filled symbols) cells \pm SEM. (C) Wound-healing migration assay. Confluent cells were induced to migrate by scratching, fixed at the indicated time-points and stained with Giemsa stain. Migration is given as percentage of wound closure (i.e., distance moved by the cells) with respect to time 0 (100\% opened wound). HER2-silenced (open symbols) and control (filled symbols) cells. Average of 4 experiments \pm SEM. (D) Trans-well migration assay. Cells were applied to the upper chamber of trans-well membranes and grown overnight. Media added with fetal calf serum (FCS) or bovine albumin (BSA) was present in the lower chamber. The migrated cells were fixed, stained with Crystal violet, lysed and analyzed spectrophotometrically. Average of 3 experiments \pm SEM. 
involvement of FAK and other cell motility molecules, confirmed this hypothesis.

\subsection{HER2 affects the expression of several PTPs and DSPs, and primarily of PTPN12}

Since several Tyrosine Phosphatases (PTPs) and Dual-Specificity Phosphatases (DSPs) play a role in the HER2-dependent signalling and might mediate the HER2-driven motility pathways, we investigated whether the expression of any of these was affected by HER2. For this purpose we adopted a custom DNA microarray, displaying all the 103 human PTPs and
DSPs (designed for a previous investigation, [8]) and analysed the changes consequent to HER2 silencing. Four PTPs and 5 DSPs were found up-regulated (23-fold), whereas DUSP4 was strongly down-regulated in HER2-silenced cells (Fig. 2A). Since RNA changes are not necessarily followed by protein changes, we analysed the protein level of all the altered PTPs and of some of the altered DSPs (guided by the availability of reliable antibodies). Only PTPN12 (PTP-PEST) was highly increased in HER2-silenced cells, with respect to control cells. Also PTPN11 (SHP2) displayed some increase, whereas PTPRK, PTPN6, PTEN and $\mathrm{CDC} 25 \mathrm{C}$ were unchanged (Fig. 2B and C). Based on the remarkable change and the involvement of
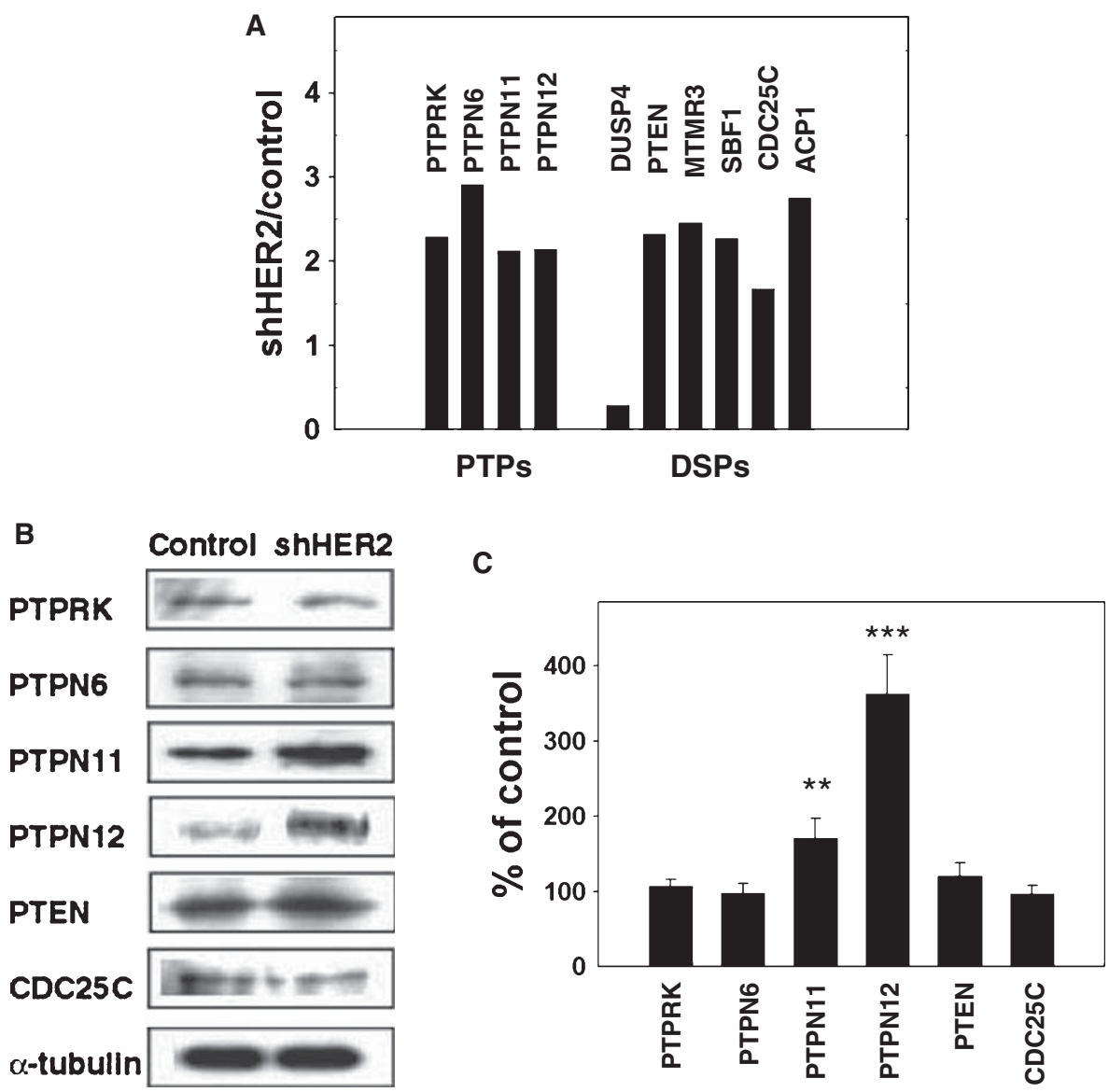

Fig. 2. Tyrosine phosphatases (PTPs) and Dual-Specificity phosphatases (DSPs) in HER2-silenced SKOV-3 cells. (A) RNA fold-change. Total RNA was purified from HER2-silenced and control cells (carrying the empty vector) and used to generate labeled antisense RNA for subsequent DNA microarray hybridization. The results are given as expression rates of shHER2/control cells. Average of 3 experiments, performed with RNA derived from independent purifications. (B) Protein immunoblots. Extracts were prepared from cells as in A and used to immunodetect the indicated PTPs and DSPs and $\alpha$-tubulin. (C) Immunoblot quantification. Protein bands as in B were quantified by densitometric scanning and normalized to $\alpha$-tubulin, detected on the same membrane. Results are given as percent of normalized bands from control cells (i.e., 100 values are equal to control values). Average \pm SEM of 3-4 independent experiments. Statistical analysis (Student's $t$ test): ${ }^{* *} p<0.01 ;{ }^{* *} p<0.001$. 
PTPN12 in migration, we set to investigate whether its HER2-driven down-modulation may sustain the motility of SKOV-3 cells.

\subsection{PRPN12 depresses cell migration}

In order to learn more about its role in SKOV-3 cells, PTPN12 was silenced. Stable silencing induced over $80 \%$ decrease in PTPN12 protein (Fig. 3A and B) and enhanced migration, whether assessed through woundhealing assay (Fig. 3C) or trans-well assay (Fig. 3D). Morphological changes, such as cell detachment and rounding, were also observed (not shown). On the other hand, PTPN12-silencing did not affect cell proliferation (not shown). The results defined PTPN12 as a negative regulator of SKOV-3 cell migration. This, in turn, may support the hypothesis that downmodulation of PTPN12 by HER2 enhances migration and contributes to the motility of SKOV-3 cells.

A control shPTPN12

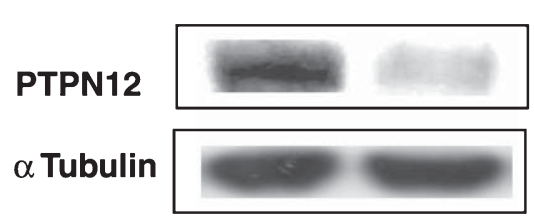

C

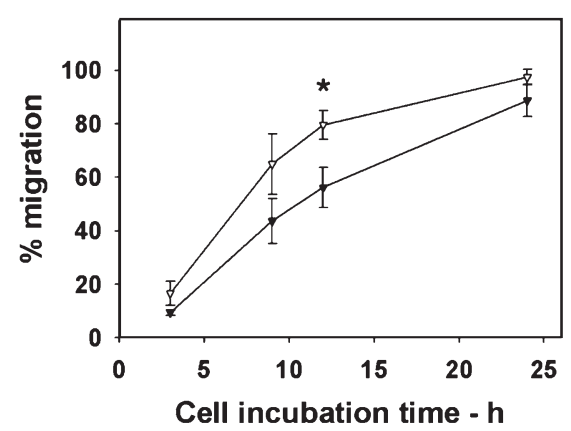

\subsection{PTPN12 affects FAK phosphorylation at Y397 and FAK activity}

To investigate the PTPN12 mechanism, we focused on FAK, a key regulator of cell adhesion and motility. In a preliminary test, we assessed the ability of PTPN12 to associate with FAK in control SKOV-3 cells. The results indicated that PTPN12 co-immunoprecipitated with FAK and within this complex PTPN12 was active, based on in-gel activity assay (Fig. 4A). The phosphorylation of the FAK autophosphorylation site Y397, a major regulator of FAK activity [26, 28], was much lower in HER2-silenced cells than in control cells (Fig. 4B), supporting the hypothesis that PTPN12 dephosphorylates Y397, thus inactivating FAK. The decrease in FAK activity was confirmed by direct kinase activity assay of immunoprecipitated FAK (Fig. 4C). On the other hand, in PTPN12-silenced cells the level of pY397 and FAK kinase activity were similar to control cells (Fig. 4B and C). This result does

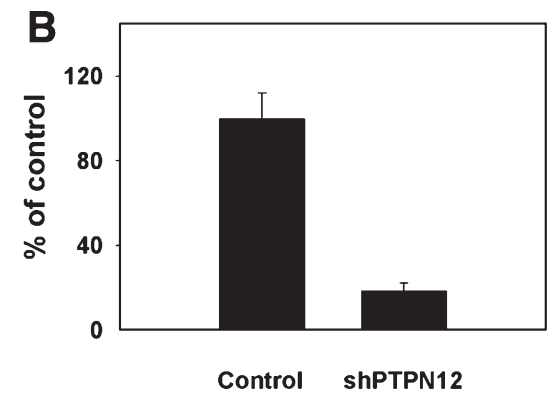

D

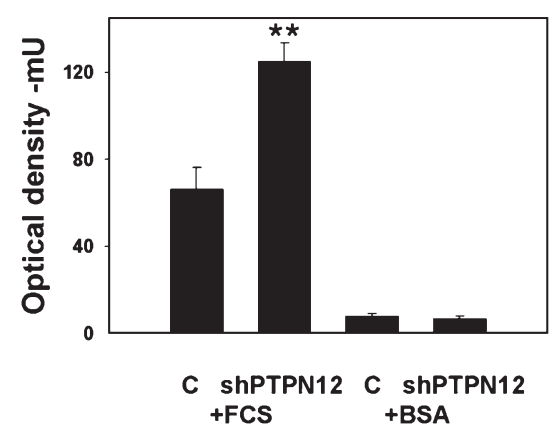

Fig. 3. PTPN12 silencing and effects on cell migration in SKOV-3 cells. (A) PTPN12 protein change. Extracts were prepared from cells carrying stable PTPN12 silencing and from control cells (carrying the empty vector) and used to immunodetect PTPN12 and $\alpha$-tubulin. (B) Quantification of PTPN12 protein change. PTPN12 protein bands as in A were quantified by densitometric scanning and normalized to $\alpha$-tubulin. Results are given as percent of normalized bands from control cells. Average \pm SEM of 3 independent experiments, using 2 different silenced clones. (C) Wound-healing migration assay. Confluent cells were induced to migrate and analyzed as in Fig. 1. PTPN12-silenced (open symbols) and control (filled symbols) cells. Average of 3 experiments \pm SEM. Statistical analysis (Student's $t$ test): ${ }^{*} p<0.05$. (D) Trans-well migration assay. The assay was performed as in Fig. 1. Average of 3 experiments \pm SEM. Statistical analysis (Student's $t$ test): ${ }^{* *} p<0.01$. 
A
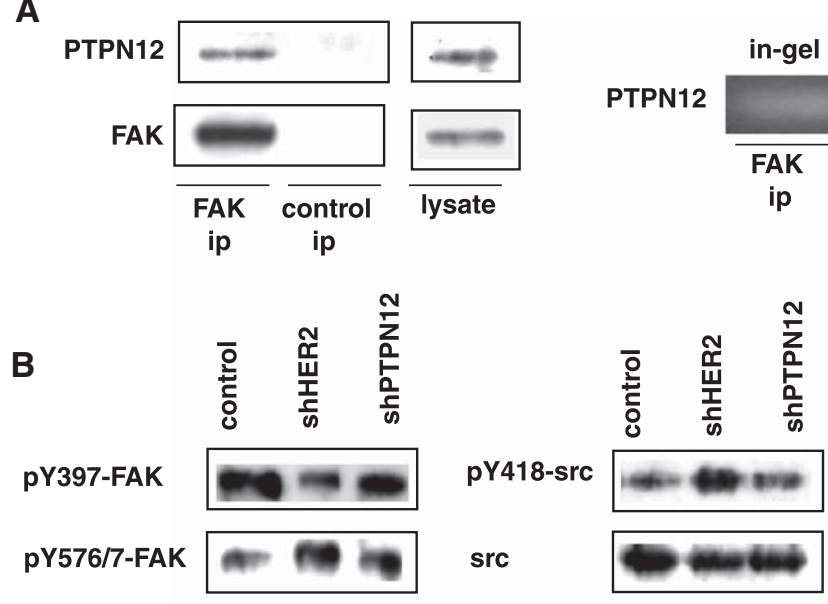

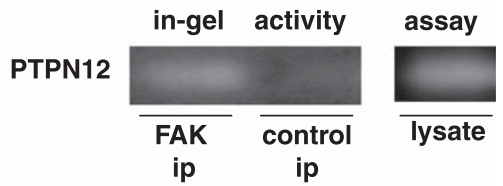

C

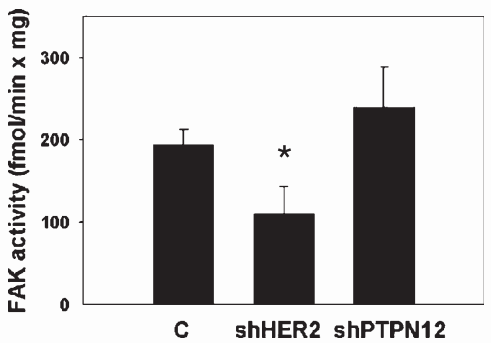

D

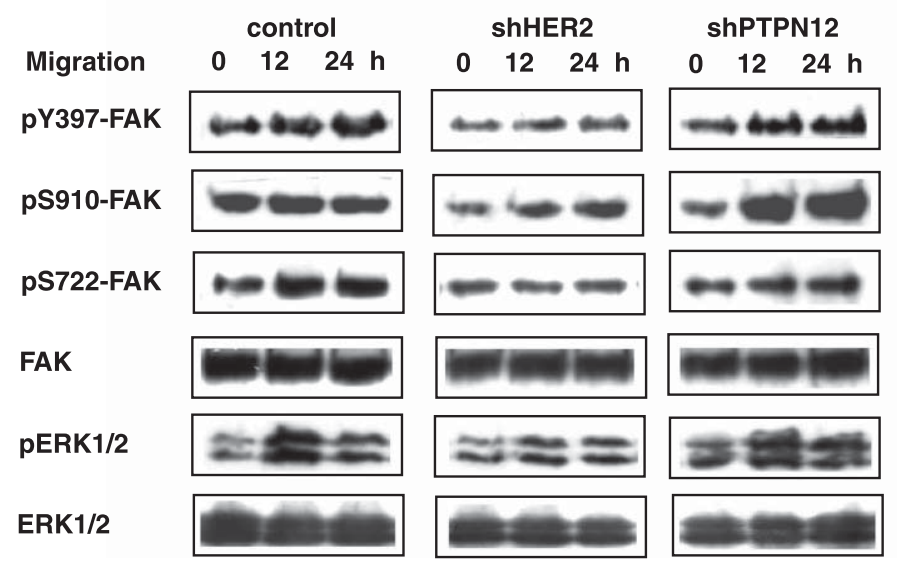

Fig. 4. FAK regulation in cells carrying silenced HER2 or silenced PTPN12. (A) FAK and PTPN12 association. Anti-FAK and control immunocomplexes were obtained form SKOV-3 cells and assayed for the presence of PTPN12 and FAK by immunoblot, and for the phosphatase activity of PTPN12 by in-gel assay (further described in the Methods section). PTPN12, FAK and PTPN12 activity detected in cell lysates are also shown. The data are representative of 3 independent experiments. (B) FAK activation. Lysates prepared from HER2-silenced and PTPN12-silenced cells as well as control cells (carrying the empty vector) were used to immunodetect the phosphorylation of FAK at Y397, Y576/577, S910 and S722, FAK, the phosphorylation of Y416 of src, src and $\alpha$ - tubulin. The data are representative of several independent experiments. (C) FAK kinase activity. FAK was immunoprecipitated from lysates as in B and the kinase activity was assayed using the substrate poly-Glu-Tyr, as further described in the Methods section. Average \pm SEM of 3 experiments. Statistical analysis (Student's $t$ test): ${ }^{*} p<0.05$. (D) FAK and ERK1/2 activation in migrating cells. Lysates prepared from cells induced to migrate in a wounded area (as in Fig. 1B) and collected at the indicated time points were used to immunodetect the phosphorylation of FAK at Y397, S910, S722 and pERK1/2 as well as FAK, ERK1/2 and $\alpha$-tubulin. The data are representative of 3 independent experiments. 
not account for the increased motility of the PTPN12silenced cells (Fig. 3C and D), hence the contribution of other FAK sites, including some analysed further on, should be taken onto account. As a potential PTPN12 target, we examined also FAK Y576/577, sites phosphorylated by src. The phosphorylation of Y576/577 was higher in HER2-silenced than in control cells, hence opposite to what one might expect for a PTPN12 target (Fig. 4B). The reliability of the result was confirmed by the concomitant activation of src, indicated by the increased src pY418 (Fig. 4B) (phosphorylation of FAK Y576/577 is considered a good indicator of src activation [45]). Conversely, in PTPN12-silenced cells pY576/577 was low, again in agreement with the detected src inactivation (low pY418, Fig. 4B). A possible explanation for src activation in spite of HER2 silencing might be that these poorly motile cells are also more adherent than control cells, a condition that justifies src activation. Altogether, the results suggested targeting of FAK Y397 by PTPN12 and excluded Y576/577.

\subsection{HER2 affects FAK phosphorylation at $\$ 910$ and $S 722$, in quiescent or migrating cells}

Several other phosphorylation sites contribute to FAK regulation in motility. In previous investigations we addressed the role of two FAK sites: S910, linked to cell shape and motility [31], and S722, responding to cell adhesion and migration [32]. S910 displayed decreased phosphorylation in HER2-silenced cells, whereas it was close to control level in PTPN12silenced cells. On the other hand, pS722 was little affected in quiescent cells (Fig. 4B).

The FAK sites were also analysed in cells induced to migrate in a wounded area. Though migration increased the phosphorylation of FAK Y397 in all cell types, the phosphorylation levels were much lower in HER2-silenced cells at all time-points. This result agrees with the data obtained in quiescent cells (Fig. 4B) and with the decreased motility displayed by HER2-silenced cells (Fig. 1C and D). Also pS910 was confirmed to be lower in HER2-silenced cells and increased in migrating HER2-silenced and PTPN12silenced cells, suggesting motility-linked regulation. On the other hand its level was constantly high in control cells, a fact that is possibly explained by the activation of ERK5 (see further on and Discussion). pS722 was constantly low in HER2-silenced cells, further stressing the migration impairment. On the other hand, pS722 increased in control and PTPN12silenced cells, confirming the increase in pS722 during late migration that we previously described in fibroblasts [32]. Finally, ERK1/2, which is stimulated during migration (see pERK1/2 in control cells), was less activated in migrating HER2-silenced cells than in control or PTPN12-silenced cell (Fig. 4D). Altogether the results indicated alteration of several phosphorylation sites that may contribute to impair migration in HER2silenced cells (displaying high PTPN12) and to sustain migration in PTPN12- silenced cells (displaying high HER2). The most intriguing changes concerned S910, a site that was further investigated.

\subsection{ERK5 targets $S 910$ of FAK in HER2-dependent way}

FAK S910 was reported to be a target for either ERK1/2 or ERK5 [31, 35]. In our system either kinase might be involved, since HER2 silencing affected ERK1/2 (Fig. 1A and 4D) and ERK5 is known to be stimulated by HER2 [40]. To discriminate between these two kinases we used the MEK inhibitor U0126. Blot analysis of lysates from SKOV-3 cells treated with 1 or $10 \mu \mathrm{M}$ U0126 indicated that ERK1/2 was completely inactivated at both inhibitor concentrations, whereas S910 was still phosphorylated (Fig. 5A). While ruling-out ERK1/2, the results suggested the involvement of ERK5, which is much less sensitive to U0126 than ERK1/2 [31, 46]. To test this hypothesis, ERK5 was silenced in SKOV-3 cells. Stable silencing was confirmed by almost complete loss of ERK5, detected as both protein and kinase activity, assayed with the ERK5-specific substrate Mef2 [31] (Fig. 5B and $\mathrm{C}$; the inset of Fig. 5B shows one set of the autoradiography data used to generate the bar-graph). Loss of pS910 in ERK5-silenced cells (Fig. 5C) clearly indicated that S910 phosphorylation was due to ERK5.

The subsequent step was aimed to assess if also the ERK5 activity was affected by HER2-silencing. The ERK5 kinase assay indicated a significant ERK5 inactivation in HER2-silenced cells (Fig. 5D). As an additional test, we adopted the alternative substrate myelin basic protein to assay the kinase activity of immunoprecipitated ERK5. The results confirmed ERK5 inactivation in HER2-silenced cells (Fig. 5D; the insets show one set of the autoradiography data used for the bar-graph). Finally, the ERK5 protein 
A

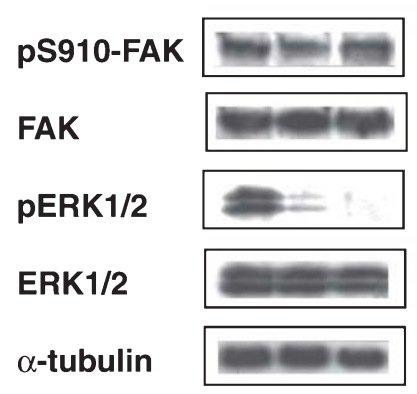

C C ShERK5

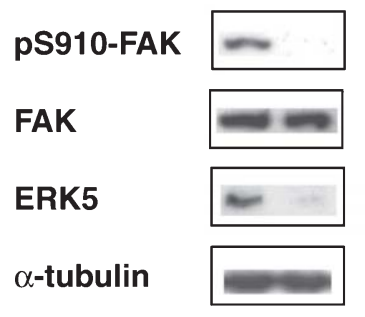

\section{B}
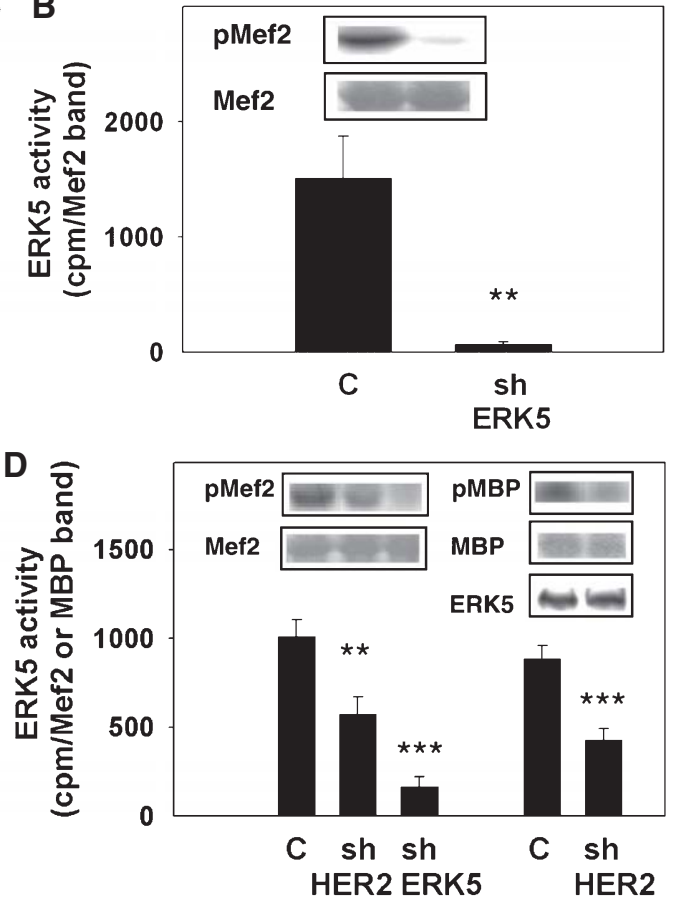

Fig. 5. ERK5 in the HER2-dependent regulation of FAK. (A) Effect of the MEK inhibitor U0126 on FAK and ERK1/2. SKOV-3 cells were treated with the indicated amounts of U0126 for $1 \mathrm{~h}$ and the lysates obtained were used to immunodetect the phosphorylation of FAK S910 and ERK1/2, FAK, ERK1/2 and $\alpha$-tubulin. The data are representative of 3 independent experiments. (B) ERK5 silencing. Lysates prepared from ERK5-silenced and control cells (carrying the empty vector) were used to assay the ERK5 kinase activity with the ERK5-specific substrate Mef2, as further described in the Methods section. Average \pm SEM of 4 experiments. Statistical analysis (Student's ttest): ${ }^{* *} p<0.01$. Inset: Autoradiographic detection of pMef2 phospshorylated with extracts from control and ERK5-silenced cells and Coomasie blue staining of GST-Mef2. The inset shows an example of the autoradiography data used to generate the bar-graph, as further described in the Methods section. (C) Effect of ERK5-silencing on the phosphorylation of FAK at S910. The phosphorylation of FAK S910, FAK, ERK5 and $\alpha$-tubulin were immunodetected in lysates as in B. The results are representative of 3 independent experiments using 2 different ERK5-silenced clones. (D) Effect of HER2-silencing on ERK5 activity. Lysates were obtained from HER2-silenced, ERK5-silenced or control SKOV-3 cells and used to assay ERK5 either as Mef2 kinase (as in B) or as Myelin Basic Protein (MBP) kinase of immunoprecipitated ERK5 (further described in the Methods section). Average \pm SEM of 4 experiments. Statistical analysis (Student's $t$ test): ${ }^{* *} p<0.01$; ${ }^{* * *} p<0.001$. Inset. Autoradiographic detection of pMef2 phosphorylated with extracts from control, HER2-silenced and ERK5-silenced cells and Coomasie blue staining of GSTMef2. Autoradiographic detection of pMBP phospshorylated by ERK5 immunoprecipitated from control and ERK5-silenced cells cell extracts, Coomassie blue staining of MBP and immunoblot detection of ERK5. The insets show examples of the autoradiography data used for the bar-graph.

level was not affected by HER2-silencing (Fig. 5D, inset), indicating that ERK5 inactivation was due to posttranslational regulation.

\section{Discussion}

Most of the studies on PTPs and DSPs in HER2 signalling focused on post-translational regulation, whereas here we investigated their expression alter- ations linked to HER2 modulation. The highly motile SKOV-3 cells were adopted to identify PTPs and DSPs potentially involved in motility pathways. The results supported the hypothesis that HER2 activation affects the expression of several PTPs and DSPs, based on the detected up-modulation of 4 PTPs (PTPK, PTPN6, PTPN11, PTPN12) and 5 DSPs (PTEN, MTMR3, SBF1, CDC25C, ACP1) and down-modulation of DUSP4 in HER2-silenced cells. Moreover, the 4 PTPs and some of the DSPs (PTEN, DUSP4, ACP1) are rel- 
evant to cell adhesion or migration, further supporting the hypothesis of their involvement in mediating the HER2-driven cell motility.

In a previous investigation, a similar analysis in human HER2 breast cancer and HER2-overexpressing MDA-MB-453 breast cancer cells identified several altered PTPs and DSPs. Among these, DUSP6, CDC25B and MTMR1 were altered in both tumours and cells, hence potentially contributing to HER2driven tumorigenesis [8]. We also proposed that DUSP6, up-modulated in breast tumours at a level comparable to ERBB2, might represent a new breast tumour marker or a potential therapy target. Comparison with the present results indicates that the altered PTPs and DSPs identified in the breast and ovary studies show only few overlaps (PTPN11, PTEN and CDC25C), which may indicate the specificity of the results obtained and suggests differential HER2 roles in the two systems.

The role of PTPN12 in motility has been studied in fibroblasts, but is much less defined in epithelial cells, though recent reports involved PTPN12 in the motility of pancreatic and intestinal carcinoma cells [22, 23]. In our system, comparison of HER2-silenced and PTPN12-silenced cells indicated almost symmetrical behaviours. HER2-silencing impaired migration and up-regulated PTPN12, whereas PTPN12-silencing (on an HER2 up-regulated background) enhanced migration and induced morphological changes, suggesting cytoskeletal rearrangements. This brought to propose that HER2-overexpression depresses PTPN12, contributing, in turn, to the marked motility of SKOV-3 cells. Dephosphorylation of FAK by PTPN12 was reported also in fibroblasts, but in this case it occurred in cells induced to migrate by activated ras [23]. On the contrary, in our system migration induced increase in Y397 phosphorylation, whereas loss of phosphate at Y397 occurred in HER2-silenced cells, which are less motile. We conclude that targeting of Y397 by PTPN12 might be a general mechanism, but the cells analysed as well as the migration cues may account for the marked differences reported by the two studies.

Analysis of FAK phosphorylation at S910 uncovered the role of ERK5 and excluded ERK1/2, although both kinases are controlled by HER2 (Fig. 1A and 5D; see also [40]). In HER2-silenced SKOV-3 cells, pS910 decreased in parallel with the decrease in ERK5 activity and FAK inactivation, suggesting the involvement of the phosphorylation of S910 by ERK5 in the control of FAK. Also the increase in pS910 during the migration of HER2-silenced and PTPN12-silenced cells suggested that this site is regulated during motility. On the other hand, in control cells pS910 was constantly high and did not change in migration. This might be explained by assuming that the high, HER2dependent ERK5 activity induced a pS910 level that could not be increased any further. An alternative possibility is the involvement of the phosphatase PP $1 \delta$ that targets pS910 [31] and associates with FAK at focal adhesions [47], but whose regulation during migration is still unknown. In a previous investigation we reported that FAK S910 was phosphorylated by ERK5 in fibroblasts and breast carcinoma cell lines. Treatment with the phorbol ester TPA increased pS910 and induced morphological changes suggesting cytoskeletal rearrangements [31]. More recently the involvement of ERK5 in adhesion and migration was indicated in keratinocytes [37] and endothelial cells [38]. Connection between ERK5 and FAK was also reported in cell adhesion to vitronectin, however indicating that ERK5 was activated downstream of FAK [36], hence depicting a mechanism different from the one described herein.

Among the altered PTPs, also PTPN11 (SHP2) displayed up-regulation and protein increase in HER2-

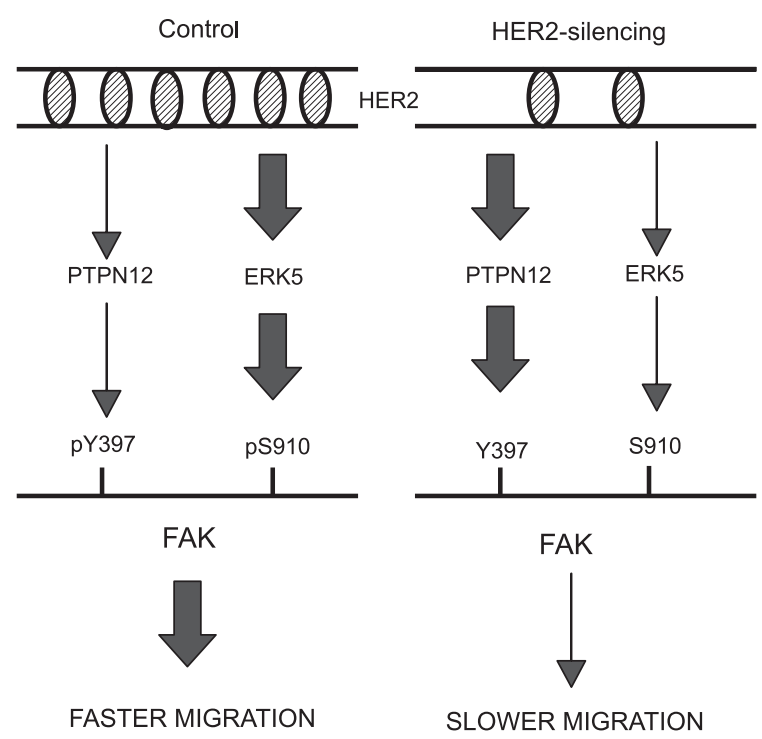

Fig. 6. Proposed contribution of PTPN12 and ERK5 to the HER2directed cell migration. FAK phosphorylation at Y397 and S910 by PTPN12 and ERK5, in control and HER2-silenced SKOV-3 cells, and consequences on FAK activity and cell migration. The arrow thickness defines low (thin arrow) or high (thick arrow) enzyme activity. 
silenced cells. PTPN11 generally transduces a positive signal and enhances ERK activation in growth factor pathways, including those evoked by HER2 (e.g., in breast cancer cells [48]). However, its role in integrating receptor and integrin signals is not completely clear due to conflicting results (e.g., with respect to FAK and src regulation [11]). Our results indicate a negative control of PTPN11 by HER2 in SKOV-3 cells, suggesting, in turn, negative control of motility and a possible cooperation with PTPN12 that would deserve further investigation.

Altogether, the results suggest the ability of PTPN12 and ERK5 to mediate the HER2-driven cell motility in SKOV-3 cells, through FAK targeting (Fig. 6). This may represent a new aspect of the complex cross-talk between HER 2 and the migration machinery required for normal cell life and tumour invasion. A better understanding of the regulation of specific genes (e.g., kinases and phosphatases) linked to enhanced motility in tumour cells, may lead to identify new tumour markers. These, in turn, might help to predict invasiveness and metastatic potential already in primary tumours. Within this picture, valuable information may come also from the analysis or generation of cell lines with varying metastatic abilities. All these issues seem to be specifically relevant in the case of cancers, including ovarian cancers, that become highly metastatic at some stages.

\section{Acknowledgments}

This work was supported by grant from AIRC (Milan, I).

\section{References}

[1] A. Wolf-Yadlin, N. Kumar, Y. Zhang, S. Hautaniemi, M. Zaman, H.D. Kim, V. Grantcharova, D.A. Lauffenburger and F.M. White, Effects of HER2 overexpression on cell signaling networks governing proliferation and migration, Mol Syst Biol 2 (2006), 54

[2] K.S. Spencer, D. Graus-Porta, J. Leng, N.E. Hynes and R.L. Klemke, ErbB2 is necessary for induction of carcinoma cell invasion by ErbB family receptor tyrosine kinases, J Cell Biol 148 (2000), 385-397.

[3] L. Zhan, B. Xiang and S. K. Muthuswamy, Controlled activation of ErbB1/ErbB2 heterodimers promote invasion of three-dimensional organized epithelia in an ErbB 1-dependent manner: implications for progression of ErbB2-overexpressing tumors, Cancer Res 66 (2006), 5201-5208.

[4] B. Linggi and G. Carpenter, ErbB receptors: new insights on mechanisms and biology, Trends Cell Biol 16 (2006), 649-656.
[5] N.E. Hynes and G. MacDonald, ErbB receptors and signaling pathways in cancer, Curr Opin Cell Biol 21 (2009), 177-184.

[6] J.N. McAlpine, K.C. Wiegand, R. Vang, B.M. Ronnett, A. Adamiak, M. Kobel, S.E. Kalloger, K.D. Swenerton, D.G. Huntsman, C.B. Gilks and D.M. Miller, HER2 overexpression and amplification is present in a subset of ovarian mucinous carcinomas and can be targeted with trastuzumab therapy, $B M C$ Cancer 9 (2009), 433.

[7] N.K. Tonks, Protein tyrosine phosphatases: from genes, to function, to disease, Nat Rev Mol Cell Biol 7 (2006), 833-846.

[8] M.A. Lucci, R. Orlandi, T. Triulzi, E. Tagliabue, A. Balsari and E. Villa-Moruzzi, Expression profile of tyrosine phosphatases in HER2 breast cancer cells and tumors, Cell Oncol 32 (2010), 361-372.

[9] M. Larsen, M.L. Tremblay and K.M. Yamada, Phosphatases in cell-matrix adhesion and migration, Nat Rev Mol Cell Biol 4 (2003), 700-711.

[10] S.M. Keyse, Dual-specificity MAP kinase phosphatases (MKPs) and cancer, Cancer Metastasis Rev 27(2008), 253-261.

[11] B.G. Neel, H. Gu and L. Pao, The 'Shp'ing news: SH2 domaincontaining tyrosine phosphatases in cell signaling, Trends Biochem Sci 28 (2003), 284-293.

[12] N.K. Tonks and S.K. Muthuswamy, A brake becomes an accelerator: PTP1B-a new therapeutic target for breast cancer, Cancer Cell 11 (2007), 214-216.

[13] S. Granot-Attas, H. Knobler and A. Elson, Protein tyrosine phosphatases in osteoclasts, Crit Rev Eukaryot Gene Expr 17 (2007), 49-71.

[14] R. Boutros, C. Dozier and B. Ducommun, The when and wheres of CDC25 phosphatases, Curr Opin Cell Biol 18(2006), $185-191$.

[15] M. Gensler, M. Buschbeck and A. Ullrich, Negative regulation of HER2 signaling by the PEST-type protein-tyrosine phosphatase BDP1, J Biol Chem 279 (2004), 12110-12116.

[16] M.G. Mohi and B.G. Neel, The role of Shp2 (PTPN11) in cancer, Curr Opin Genet Dev 17 (2007), 23-30.

[17] M. Bentires-Alj and B.G. Neel, Protein-tyrosine phosphatase $1 \mathrm{~B}$ is required for HER2/Neu-induced breast cancer, Cancer Res 67 (2007), 2420-2424.

[18] A.J. Garton and N.K. Tonks, Regulation of fibroblast motility by the protein tyrosine phosphatase PTP-PEST, J Biol Chem 274 (1999), 3811-3818.

[19] A. Angers-Loustau, J.F. Cote, A. Charest, D. Dowbenko, S. Spencer, L.A. Lasky and M.L. Tremblay, Protein tyrosine phosphatase-PEST regulates focal adhesion disassembly, migration, and cytokinesis in fibroblasts, J CellBiol 144 (1999), 1019-1031.

[20] S.K. Sastry, P.D. Lyons, M.D. Schaller and K. Burridge, PTPPEST controls motility through regulation of Rac1, J CellSci 115 (2002), 4305-4316.

[21] M. Halle, Y.C. Liu, S. Hardy, J.F. Theberge, C. Blanchetot, A. Bourdeau, T.C. Meng and M.L. Tremblay, Caspase- 3 regulates catalytic activity and scaffolding functions of the protein tyrosine phosphatase PEST, a novel modulator of the apoptotic response, Mol Cell Biol 27 (2007), 1172-1190.

[22] R. Espejo, W. Rengifo-Cam, M.D. Schaller, B.M. Evers and S.K. Sastry, PTP-PEST controls motility, adherens junction assembly, and Rho GTPase activity in colon cancer cells, Am J Physiol CellPhysiol 299 (2010), C454-C463. 
[23] Y. Zheng, Y. Xia, D. Hawke, M. Halle, M.L. Tremblay, X. Gao, X.Z. Zhou, K. Aldape, M.H. Cobb, K. Xie, J. He and Z. Lu, FAK phosphorylation by ERK primes ras-induced tyrosine dephosphorylation of FAK mediated by PIN1 and PTP-PEST, Mol Cell 35 (2009), 11-25.

[24] M.A. Chellaiah and M.D. Schaller, Activation of Src kinase by protein-tyrosine phosphatase-PEST in osteoclasts: comparative analysis of the effects of bisphosphonate and protein-tyrosine phosphatase inhibitor on Src activation in vitro, J CellPhysiol 220 (2009), 382-393.

[25] S. Mathew, S.P. George, Y. Wang, M.R. Siddiqui, K. Srinivasan, L. Tan and S. Khurana, Potential molecular mechanism for c-Src kinase-mediated regulation of intestinal cell migration, J Biol Chem 283 (2008), 22709-22722.

[26] S.K. Hanks, L. Ryzhova, N.Y. Shin and J. Brabek, Focal adhesion kinase signaling activities and their implications in the control of cell survival and motility, Front Biosci 8 (2003), d982-d996.

[27] S.K. Mitra, D.A. Hanson and D.D. Schlaepfer, Focal adhesion kinase: in command and control of cell motility, Nat Rev MolCell Biol 6 (2005), 56-68.

[28] J.T. Parsons, Focal adhesion kinase: the first ten years, J Cell Sci 116 (2003), 1409-1416.

[29] G.W. McLean, N.O. Carragher, E. Avizienyte, J. Evans, V.G. Brunton and M.C. Frame, The role of focal-adhesion kinase in cancer - a new therapeutic opportunity, Nat Rev Cancer 5 (2005), 505-515.

[30] A. Carlucci, C. Gedressi, L. Lignitto, L. Nezi, E. Villa-Moruzzi, E. V. Avvedimento, M. Gottesman, C. Garbi and A. Feliciello, Protein-tyrosine phosphatase PTPD1 regulates focal adhesion kinase autophosphorylation and cell migration, J Biol Chem 283 (2008), 10919-10929.

[31] E. Villa-Moruzzi, Targeting of FAK Ser910 by ERK5 and PP1 delta in non-stimulated and phorbol ester-stimulated cells, Biochem J 408 (2007), 7-18.

[32] M. Bianchi, S. De Lucchini, O. Marin, D.L. Turner, S.K. Hanks and E. Villa-Moruzzi, Regulation of FAK Ser-722 phosphorylation and kinase activity by GSK3 and PP1 during cell spreading and migration, Biochem J 391 (2005), 359-370.

[33] M. Katz, I. Amit and Y. Yarden, Regulation of MAPKs by growth factors and receptor tyrosine kinases, Biochim Biophys Acta 1773 (2007), 1161-1176.

[34] P.R. Grigera, E.D. Jeffery, K.H. Martin, J. Shabanowitz, D.F. Hunt and J.T. Parsons, FAK phosphorylation sites mapped by mass spectrometry, J CellSci 118 (2005), 4931-4935.

[35] I. Hunger-Glaser, R.S. Fan, E. Perez-Salazar and E. Rozengurt, PDGF and FGF induce focal adhesion kinase
(FAK) phosphorylation at Ser-910 : dissociation from Tyr-397 phosphorylation and requirement for ERK activation, $J$ Cell Physiol 200 (2004), 213-222.

[36] R.S. Sawhney, W. Liu and M.G. Brattain, A novel role of ERK5 in integrin-mediated cell adhesion and motility in cancer cells via Fak signaling, J Cell Physiol 219 (2009), 152-161.

[37] V. Arnoux, M. Nassour, A. L'Helgoualc'h, R.A. Hipskind and P. Savagner, Erk5 controls Slug expression and keratinocyte activation during wound healing, Mol Biol Cell 19 (2008), 4738-4749.

[38] D. Spiering, M. Schmolke, N. Ohnesorge, M. Schmidt, M. Goebeler, J. Wegener, V. Wixler and S. Ludwig, MEK5/ERK5 signaling modulates endothelial cell migration and focal contact turnover, J Biol Chem 284 (2009), 24972-24980.

[39] X. Wang and C. Tournier, Regulation of cellular functions by the ERK5 signalling pathway, Cell Signal 18 (2006), 753-760.

[40] A. Esparis-Ogando, E. Diaz-Rodriguez, J.C. Montero, L. Yuste, P. Crespo and A. Pandiella, Erk5 participates in neuregulin signal transduction and is constitutively active in breast cancer cells overexpressing ErbB2, Mol Cell Biol 22 (2002), 270-285.

[41] E. Villa-Moruzzi, Activation of the cde25C phosphatase in mitotic HeLa cells, Biochem Biophys Res Commun 196 (1993), 1248-1254.

[42] T.C. Meng, T. Fukada and N.K. Tonks, Reversible oxidation and inactivation of protein tyrosine phosphatases in vivo, Mol Cell 9 (2002), 387-399.

[43] K. Burridge and A. Nelson, An in-gel assay for protein tyrosine phosphatase activity: detection of widespread distribution in cells and tissues, Anal Biochem 232 (1995), 56-64.

[44] M. Fresu, M. Bianchi, J.T. Parsons and E. Villa-Moruzzi, Cellcycle-dependent association of protein phosphatase 1 and focal adhesion kinase, Biochem J 358 (2001), 407-414.

[45] E. Ciccimaro, S.K. Hanks and I.A. Blair, Quantification of focal adhesion kinase activation loop phosphorylation as a biomarker of Src activity, Mol Pharmacol 75 (2009), 658-666.

[46] N. Mody, J. Leitch, C. Armstrong, J. Dixon and P. Cohen, Effects of MAP kinase cascade inhibitors on the MKK5/ERK5 pathway, FEBS Lett $\mathbf{5 0 2}$ (2001), 21-24.

[47] E. Villa-Moruzzi, M. Tognarini, G. Cecchini and P.C. Marchisio, Protein phosphatase 1 delta is associated with focal adhesions, Cell Adhes Commun 5 (1998), 297-305.

[48] X. Zhou and Y.M. Agazie, Molecular mechanism for SHP2 in promoting HER2-induced signaling and transformation, $J$ Biol Chem 284 (2009), 12226-12234. 


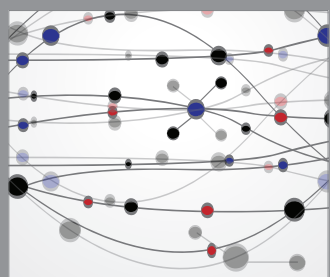

The Scientific World Journal
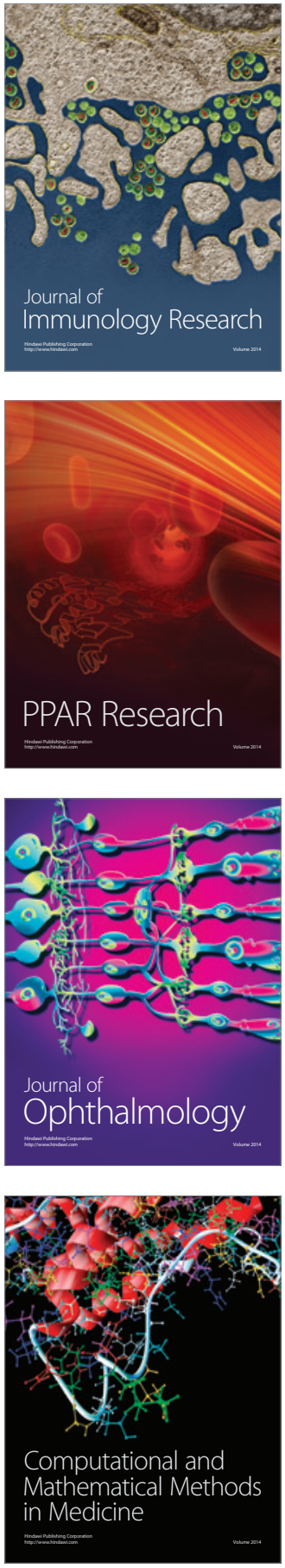

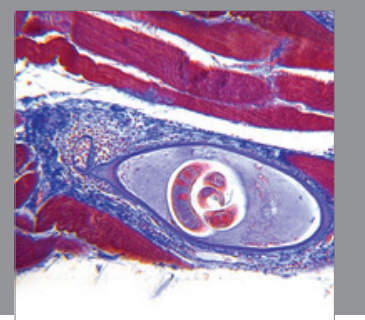

Gastroenterology

Research and Practice
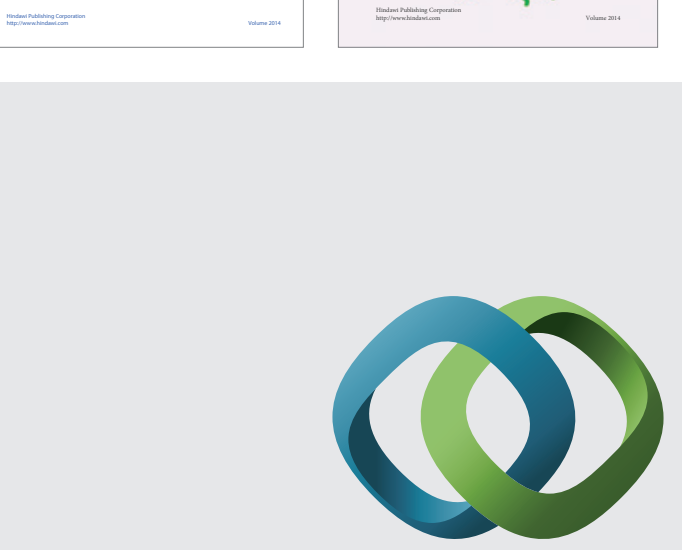

\section{Hindawi}

Submit your manuscripts at

http://www.hindawi.com
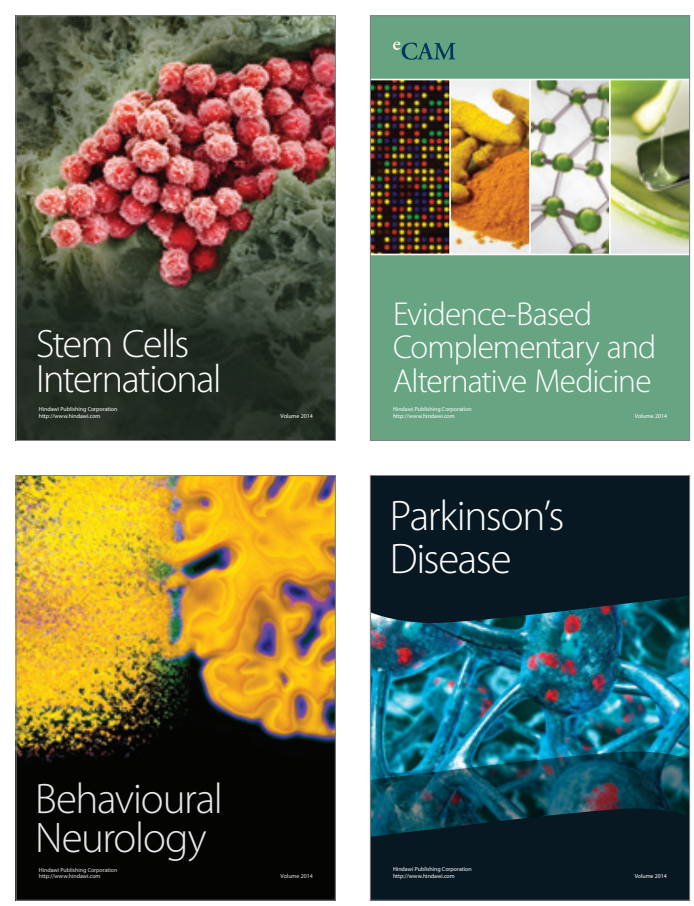

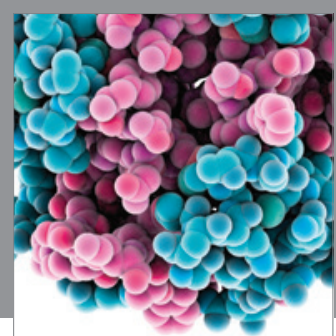

Journal of
Diabetes Research

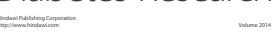

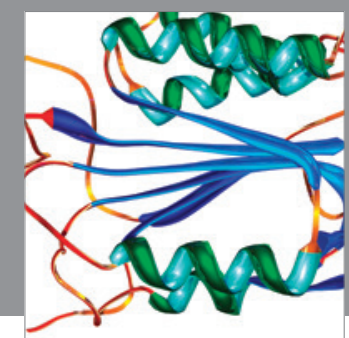

Disease Markers
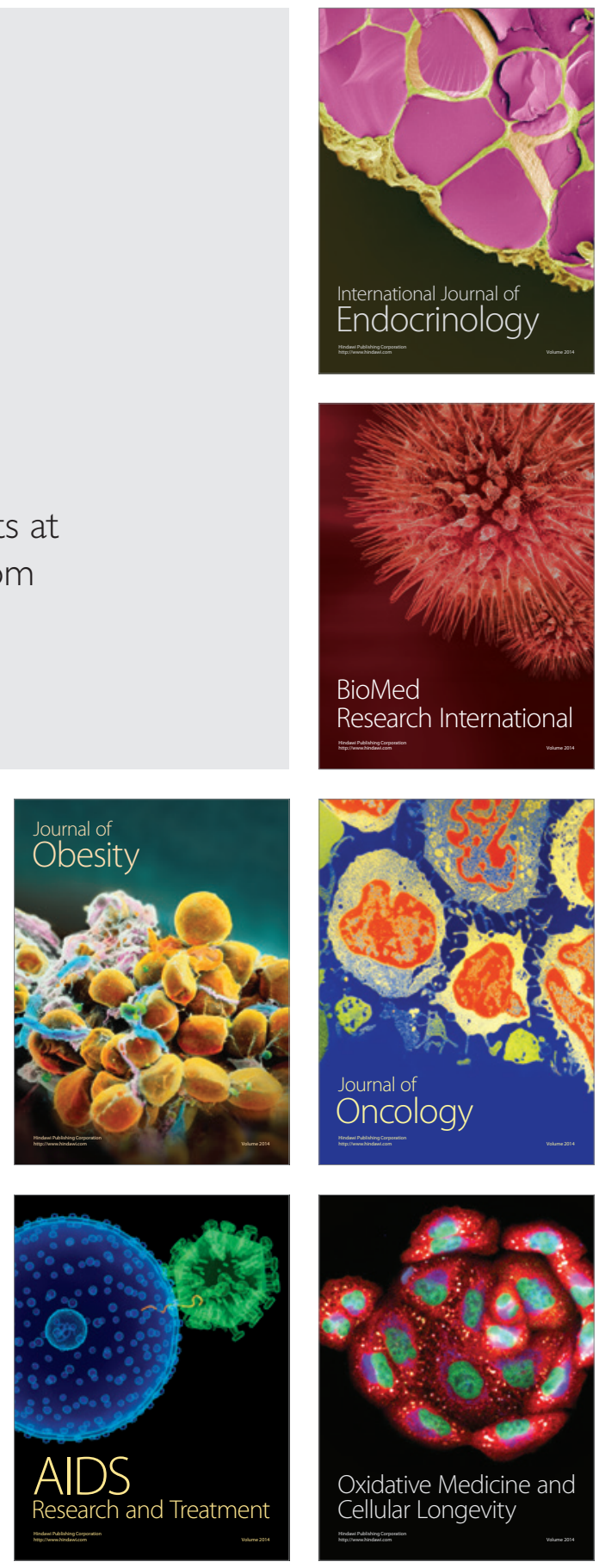